\title{
Guillain-Barre syndrome in pregnancy- a rare entity
}

\section{Snehal W. Pakhale*, Angela Sehra, Seema Bhardwaj}

Department of Obstetrics and Gynecology, Maharaja Agrasen Hospital, Punjabi Bagh West, New Delhi, India

Received: 20 August 2020

Accepted: 29 September 2020

\section{*Correspondence:}

Dr. Snehal W. Pakhale,

E-mail: drsnehalpakhale@gmail.com

Copyright: (C) the author(s), publisher and licensee Medip Academy. This is an open-access article distributed under the terms of the Creative Commons Attribution Non-Commercial License, which permits unrestricted non-commercial use, distribution, and reproduction in any medium, provided the original work is properly cited.

\begin{abstract}
Guillain-Barre syndrome (GBS) is a rare, autoimmune disorder. Estimated incidence is 0.62 to 2.66 cases per 100000 people annually. We report a case of GBS in 29 years old primigravida with pre-gestational diabetes mellitus controlled on insulin, who presented at 7 weeks of the period of gestation with complaints of numbness in both hands and fingers. After 3 days of hospital admission, she had progressive weakness in both limbs and difficulty in walking. MRI of the brain and spinal cord done to exclude other possible causes and was normal. All the other blood investigations including electrolytes were normal. Nerve conduction velocity test was suggestive of acute inflammatory demyelinating axonal motor polyneuropathy and diagnosis of GBS was made. She was managed by multidisciplinary approach in intensive care unit. The patient improved after plasmapheresis and supportive measures as thromboembolism prophylaxis and physiotherapy. Patient was discharged after 2 weeks of hospital stay and had no residual symptoms after 1 month. She was readmitted at 32 weeks of gestation with severe preeclampsia and underwent emergency cesarean section delivering $1.9 \mathrm{~kg}$ male baby. She was discharged on day 4 of cesarean section and followed in postpartum period for complications. To summarize, GBS can appear at any stage of pregnancy \& postpartum. Diagnosis can be delayed, so needs high index of clinical suspicion. Early diagnosis along with prompt intensive multidisciplinary supportive care improves outcomes for the mother and foetus.
\end{abstract}

Keywords: Guillain-Barre syndrome, Pregnancy, Brighton criteria, Plasmapheresis

\section{INTRODUCTION}

Guillain-Barré syndrome (GBS) is heterogeneous group of immune mediated peripheral neuropathies, characterized by progressive, ascending paralysis and areflexia with or without abnormal sensory function. Symptoms are preceded by an antecedent event in about two-thirds of patients. ${ }^{1}$ The disease has been linked to bacterial and viral infections, systemic diseases, neoplasia, pregnancy, traumatic injury, and organ transplant. $^{2}$ The diagnostic criteria for GBS are well defined by Brighton criteria. ${ }^{3}$

GBS can occur in any trimester of pregnancy and postpartum period but particularly in third trimester and first 2 weeks post-partum. GBS is known to worsen in postpartum period due to an increase in delayed type of hypersensitivity. ${ }^{4}$ Delayed diagnosis is also common in pregnancy or immediate postpartum period because the initial non-specific symptoms may mimic changes in the pregnancy or can be mistaken for a psychological complaint. $^{4}$

GBS has been considered a devastating disease because of its unusual and sudden onset. About one-third of the patients with GBS will require mechanical ventilation and most GBS-related deaths occur as a result of respiratory failure. In the developing world, where health facilities are limited, GBS has been shown to be an important cause of flaccid paralysis. ${ }^{2}$ 
There is no specific therapy for GBS; however, plasmapheresis and intra-venous immunoglobulin (IVIG) administration have been shown to reduce the progression and severity of disease; in fact, it has been found to accelerate the recovery of these patients. ${ }^{5}$ Reports of treatment of GBS complicating pregnancy with IVIG and plasmapheresis are available in literature. ${ }^{2}$ Our case is unique as we treated our patient in intensive care unit (ICU) with plasmapheresis in her early pregnancy (first trimester) period.

Herein, we report a patient with GBS who presented in the first trimester of pregnancy which is rare and needs high index of suspicion for its diagnosis. GBS during pregnancy, which if missed can be detrimental for the mother and foetus, was managed by multidisciplinary approach with help of gynaecologist, physician and physiotherapist. There are very few reported cases in the literature of GBS in pregnancy with different approach of management. So, we wish to report to the medical fraternity that such an event could occur anytime in pregnancy and can be managed with plasmapheresis therapy.

\section{CASE REPORT}

A 29 years old female, married since 2 years, came to obstetrics and gynaecology department of our hospital at 7 weeks period of gestation (spontaneous conception), with 1 day history of numbness in both hands and fingers. Detailed history of patient revealed that she was pregestational diabetic on insulin therapy and with deranged blood sugar levels as fasting blood sugar level was $199 \mathrm{mg} / \mathrm{dl}$, postprandial blood sugar level was $276 \mathrm{mg} / \mathrm{dl}$ and HBA1C was $11.3 \%$.

On admission, she was conscious and alert, hemodynamically stable, and with no signs of respiratory distress. With the help of endocrinologist, blood sugar levels were controlled on human actrapid and isophane insulin. After 3 days, when she got up in the morning, she noticed weakness in both the limbs. The weakness worsened the following day leading to difficulty in walking. On neurological examination, she was conscious and alert but restless and anxious. All her cranial nerves were intact. There was no sign of meningism. She had flaccid quadriparesis with grade 3 power in both lower limbs and grade 4 in upper limbs. Hypotonia was seen in both lower limbs, diminished bilateral ankle reflexes, and both knee joint reflexes were preserved. Sensory system was normal, and bladder and bowel were not involved. The neurologist was invited who made a diagnosis of GBS based on the history and findings on physical examination. Further investigations were arranged to rule out other possibilities.

Blood investigations including full blood count, serum electrolytes, serum creatinine, serum calcium, serum magnesium, and creatinine phosphokinase-MB remained within normal limits. Electrocardiogram revealed sinus tachycardia. Magnetic resonance imaging of the brain and spinal cord was found to be normal. Nerve conduction velocity study showed features of acute inflammatory demyelinating axonal motor polyneuropathy suggestive of GBS. Ultrasound scan confirmed fetal viability.

Patient was managed in intensive care unit with multidisciplinary approach. The next day plasmapheresis was started; this was the 6th day of illness. Therapeutic plasmapheresis was performed as first line treatment. Plasmapheresis was done on alternate days, with a total of three sessions and in each session; typically, 30-40 $\mathrm{mL} / \mathrm{kg}$ of plasma (1-1.5 times of plasma volumes) was removed at each procedure and five percent albumin was used as replacement fluid. No plasma was used as the patient's coagulation profile was within normal limits. Peripheral femoral access was used to carry out the procedure; proper catheter care was maintained and lines were flushed with heparin before and after the exchange.

The patient had no complications during these sessions. Complete coagulation profile including: prothrombin time (PT), activated partial thromboplastin time (APTT); complete blood count (CBC) including haemoglobin, platelet count; electrolytes $(\mathrm{Na}+, \mathrm{K}+, \mathrm{Ca}++)$; serum protein levels were checked before and after the procedure and remained within normal limits throughout the treatment period. After three sessions of plasmapheresis, i.e. on the 10th day of illness, physiotherapy and thromboprophylaxis were started simultaneously. Close observation of symptoms and signs done, over next 72 hours response as muscle tone, power, reflexes and sense of well-being was noted. The patient was discharged on 14th day of illness. At the time of discharge, the power was 5/5 in upper limbs and 3/5 in the lower limbs. Follow-up of the patient after 1 month showed no residual weakness.

Patient was readmitted at 32 weeks with severe preeclampsia for which emergency caesarean section was done, delivering male baby of $1.9 \mathrm{~kg}$ weight who was managed in neonatal ICU. Postoperatively, patient was stable with no neurological symptoms, so was discharged on 4th day of caesarean section. Follow-up of the patient after 2 weeks and 6 weeks for complications or relapse of GBS done showing patient being normotensive but with diabetes mellitus. Baby is alive and healthy with no anomalies.

\section{DISCUSSION}

GBS has a very low incidence during pregnancy. Estimated population incidence ranges from 0.62 to 2.66 cases per 100,000 person-years across all age groups. ${ }^{6}$ Its occurrence can be established as $13 \%$ in the first trimester, $47 \%$ in the second trimester, and $40 \%$ in the third trimester. ${ }^{7}$ It is usually preceded by a bacterial or viral infection. ${ }^{8}$ It has also been linked to underlying systemic diseases, certain malignancies, surgery, pregnancy, trauma, severe infection, and tissue 
transplantation; acute cytomegalovirus infection complicating twin pregnancy has been reported. GBS classically presents with pain, numbness, paresthesia, or weakness of the limbs and this can be mistaken for a psychological complaint or to some neurological diseases or often confused with pregnancy symptoms leading to delay in diagnosis and treatment.

The diagnosis of GBS is based on descriptive clinical, laboratory, and electro-diagnostic criteria. There are no pathognomonic, clinical features, and at present, no biomarkers are available to discriminate from disorders resembling GBS. ${ }^{3}$ Our patient did not give permission for lumbar puncture, so CSF was missing for diagnosis. But, NCV study was consistent with finding of GBS.

The management of GBS in pregnancy is somewhat similar to that in the non-pregnant population which includes IVIG, plasmapheresis, physiotherapy and ventilator support wherever required. Intravenous immunoglobulin and plasmapheresis are so far standard treatment for GBS, but data regarding its use in pregnancy are limited. ${ }^{9-11}$ Immunomodulation with plasmapheresis and IVIG had been found to improve treatment outcomes with full recovery in $70-80 \%$ of patients. $^{2}$ Mehndiratta et al described the efficacy and cost-effectiveness of current therapies in GBS. ${ }^{12}$ Cost of plasmapheresis is very less compared with IVIG with similar results. Hence, plasmapheresis was used as first choice of treatment in our patient.

It has been known that the major complications of plasmapheresis in pregnancy include coagulation abnormalities, maternal sepsis, hepatitis, and hemodynamic alterations that possibly compromise uteroplacental perfusion. ${ }^{13,14}$ But, such complications did not occur in our patient after plasmapheresis.

On the other hand, the efficacy and safety of IVIG treatment has been broadly accepted in the field of reproduction such as in idiopathic thrombocytopenic purpura complicating pregnancy, recurrent spontaneous abortion and neonatal passive immune thrombocytopenia. However, if conditions need a combined therapy of plasmapheresis and IVIG, plasmapheresis should precede IVIG therapy. ${ }^{9}$ To further confirm the efficacy of IVIG, as a single treatment in GBS complicated pregnancy, prospective clinical trial comparing it with plasmapheresis are needed.

Clinical trials indicate that plasmapheresis is more effective than supportive treatment alone in reducing the median time taken for patients to recover. Available literature shows treatment of acute inflammatory demyelinating polyneuropathy (AIDP) in pregnancy with IVIG and plasmapheresis. Also, Goyal et al have described the management of a primigravida presenting at 26 weeks' gestation with plasmapheresis. ${ }^{9,15-18}$
Our patient who presented and diagnosed with GBS in first trimester of pregnancy was treated in ICU with plasmapheresis therapy, showing remarkable recovery with the treatment. In our patient's pregnancy period, there were no fetal complications, but she developed severe preeclampsia at 32 weeks for which emergency caesarean section was done, with no postpartum complications or relapse of GBS.

Poor prognostic factors for GBS are serious deficits in muscular balance, the need for assisted ventilation and reduced amplitude of evoked motor potential.19 Relapse in GBS has been reported to occur in 5.5-6.8\% of patients. ${ }^{20} \mathrm{Up}$ to $20 \%$ of patients are disabled after 1 year, and a maternal mortality of $7 \%$ has been quoted (nonpregnant GBS has mortality $<5 \%)$. $^{21}$

\section{CONCLUSION}

GBS rarely complicates pregnancy but is associated with co-morbidities. It can appear at any stage of pregnancy \& postpartum. So, obstetricians should have a high index of suspicion for early diagnosis of the GBS complicated pregnancy and treatment should be in intensive care unit with multidisciplinary approach involving ventilator support if required, plasmapheresis or IVIG, prophylaxis for venous thromboembolism, proper nutrition, infection control and psychological support should be used to improve the prognosis for both mother and fetus. However, these are not readily available in resourcelimited settings because of cost and the recovery course can be very long, requiring prolonged periods of intubation and despite best efforts maternal mortality can occur.

\section{Funding: No funding sources \\ Conflict of interest: None declared \\ Ethical approval: Not required}

\section{REFERENCES}

1. Winer JB, Hughes RA, Anderson MJ, Jones DM, Kangro H, Watkins RP. A prospective study of acute idiopathic neuropathy. II. Antecedent events. J Neurol Neurosurg Psychiatry. 1988;51(5):613-8.

2. Vasudev R, Raina TR. A rare case of Guillain-Barré syndrome in pregnancy treated with plasma exchange. Asia J Transfus Scien. 2014;8(1):59.

3. Fokke C, van den Berg B, Drenthen J, Walgaard C, van Doorn PA, Jacobs BC. Diagnosis of GuillainBarré syndrome and validation of Brighton criteria. Brain J Neurol. 2014;137(Pt 1):33-43.

4. Sharma SR, Sharma N, Masaraf H, Singh SA. Guillain-Barré syndrome complicating pregnancy and correlation with maternal and fetal outcome in North Eastern India: A retrospective study. Ann Indian Acad Neurol. 2015;18(2):215.

5. Meena AK, Khadilkar SV, Murthy JMK. Treatment guidelines for Guillain-Barré Syndrome. Ann Indian Acad Neurol. 2011;14(1):S73-81. 
6. Fernando T, Ambanwala A, Ranaweera P, Kaluarachchi A. Guillain-Barré syndrome in pregnancy: A conservatively managed case. J Fam Med Prim Care. 2016 ;5(3):688.

7. Chan LY-S, Tsui MH-Y, Leung TN. Guillain-Barré syndrome in pregnancy. Acta Obstet Gynecol Scand. 2004;83(4):319-25.

8. Hahn AF. Guillain-Barré syndrome. Lance. 1998;352(9128):635-41.

9. Yamada H, Noro N, Kato EH, Ebina Y, Cho K, Fujimoto S. Massive intravenous immunoglobulin treatment in pregnancy complicated by GuillainBarré Syndrome. Eur J Obstet Gynecol Reprod Biol. 2001;97(1):101-4.

10. Van der Meché FG, Schmitz PI. A randomized trial comparing intravenous immune globulin and plasma exchange in Guillain-Barré syndrome. Dutch Guillain-Barré Study Group. N Engl J Med. 1992;326(17):1123-9.

11. Hurley TJ, Brunson AD, Archer RL, Lefler SF, Quirk JG. Landry Guillain-Barré Strohl syndrome in pregnancy: report of three cases treated with plasmapheresis. Obstet Gynecol. 1991;78(2):482-5.

12. 1Mehndiratta MM, Chowdhury D, Goel V. Efficacy and cost effectiveness of current therapies in Guillain Barre syndrome. J Assoc Physic Ind. 2001;49:45969.

13. Wing EJ, Bruns FJ, Fraley DS, Segel DP, Adler S. Infectious Complications With Plasmapheresis in Rapidly Progressive Glomerulonephritis. J Americ Medic Assoc. 1980;28;244(21):2423-6.

14. Caudle MR, Scott JR. The Potential Role of Immunosuppression, Plasmapheresis, and
Desensitization as Treatment Modalities For $\mathrm{Rh}$ Immunization. Clin Obstet Gynecol. 1982;25(2):313-9.

15. Breuer GS, Morali G, Finkelstein Y, Halevy J. A pregnant woman with hepatitis A and Guillain-Barré. J Clin Gastroenterol. 2001;32(2):179-80.

16. Clifton ER. Guillain-Barré syndrome, pregnancy, and plasmapheresis. J Am Osteopath Assoc. 1992;92(10):1279-82.

17. Zeeman GG. A case of acute inflammatory demyelinating polyradiculoneuropathy in early pregnancy. Am J Perinatol. 2001;18(4):213-5.

18. VG. Acute inflammatory demyelinating polyneuropathy in patients with pregnancy. Neurol Ind. 2004;52(2):283.

19. Bahadur A, Gupta N, Deka D, Mittal S. Successful maternal and fetal outcome of Guillain-Barré syndrome complicating pregnancy. Indian J Med Sci. 2009;63:517-8.

20. Meenakshi-Sundaram S, Swaminathan K, Karthik SN, Bharathi S. Relapsing Guillain-Barre syndrome in pregnancy and postpartum. Ann Indian Acad Neurol. 2014;17(3):352-4.

21. Zafar MSH, Naqash MM, Bhat TA, Malik GM. Guillain-Barré Syndrome in Pregnancy: An Unusual Case. J Fam Med Prim Care. 2013;2(1):90-1.

Cite this article as: Pakhale SW, Sehra A, Bhardwaj S. Guillain-Barre syndrome in pregnancy- a rare entity. Int J Reprod Contracept Obstet Gynecol 2020;9:4734-7. 